\title{
Craniectomia descompressiva no acidente vascular encefálico de fossa posterior: revisão narrativa
}

\author{
Decompressive craniectomy in posterior fossa stroke: narrative review
}

\section{Craniectomía descompresiva en el ictus de la fosa posterior: revisión narrativa}

Amanda Brandão Lopes ${ }^{1 *}$, Gustavo Henrique Silva Sidrim¹, Haroldo Souza e Silva², Marina da Costa Lopes $^{3}$, Letícia Maria Barbosa Pinto ${ }^{1}$, Larissa Lima Pessoa de Mendonça ${ }^{4}$, Denis da Silva Guedes $^{5}$, Lara Ferreira Camacho ${ }^{6}$, Maria Eduarda Araújo Tomaz de Lima7, Gustavo de Freitas Lobato $^{8}$.

\section{RESUMO}

Objetivo: Abordar a realização da craniectomia descompressiva como tratamento na ocorrência de Acidente Vascular Encefálico (AVE) isquêmico de fossa posterior. Revisão bibliográfica: O AVE isquêmico de fossa posterior ocorre quando há obstrução arterial em uma ou mais artérias que compõem a circulação posterior cerebral, impedindo o fluxo de sangue e oxigênio adequados para a região, causado morte tecidual. Trata-se de uma condição que apresenta alto grau de complicação e elevadas taxas de morbimortalidade se não forem realizadas intervenções precoces. Entre as complicações mais comuns estão aquelas que acontecem decorrentes do processo inflamatório desencadeado pela área infartada, ganhado destaque a formação de edema. A hidrocefalia obstrutiva, seguida decompressão de tronco encefálico com consequente morte encefálica é o desfecho mais temido dessa condição médica. Considerações finais: A craniectomia descompressiva constitui uma técnica cirúrgica capaz de reduzir os efeitos intracranianos hipertensivos de forma eficaz, liberando espaço para o edema cerebral formado após a isquemia, sendo capaz de melhorar a sobrevida funcional em pacientes vítimas desse raro subtipo de AVE.

Palavras-chave: Acidente vascular encefálico, Isquemia, Fossa posterior, Craniectomia descompressiva.

\begin{abstract}
Objective: To address the performance of decompressive craniectomy as a treatment in the occurrence of Ischemic Cerebrovascular Accident (ICA) of the posterior fossa. Bibliographic review: Posterior fossa ischemic stroke occurs when there is arterial obstruction in one or more arteries that make up the posterior cerebral circulation, preventing the flow of adequate blood and oxygen to the region, causing tissue death. It is a condition that presents a high degree of complications and high morbidity and mortality rates if early interventions are not performed. Among the most common complications are those that occur as a result of the inflammatory process triggered by the infarcted area, especially the formation of edema. Obstructive hydrocephalus, followed by brainstem compression with ensuing brain death, is the most feared outcome of this medical condition. Final considerations: Decompressive craniectomy is a surgical technique capable of effectively reducing intracranial hypertensive effects, freeing space for the cerebral edema formed after ischemia, being able to improve functional survival in patients who are victims of this rare subtype of stroke.
\end{abstract}

Key words: Stroke, Ischemia, Posterior fossa, Decompressive craniectomy.

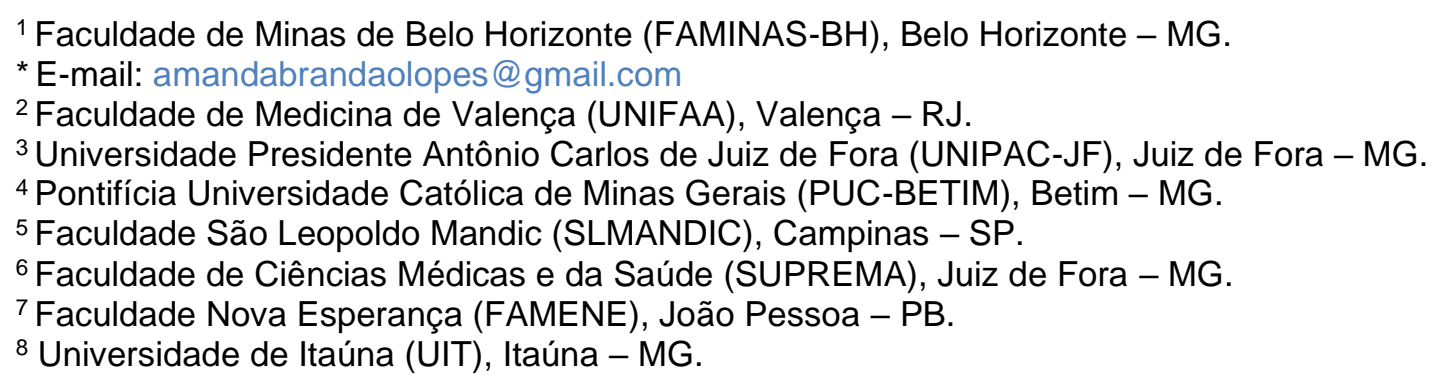




\section{RESUMEN}

Objetivo: Abordar la realización de craniectomía descompresiva como tratamiento en la ocurrencia de Accidente Cerebrovascular Isquémico (ACV) de fosa posterior. Revisión bibliográfica: El ictus isquémico de la fosa posterior ocurre cuando existe una obstrucción arterial en una o más arterias que componen la circulación cerebral posterior, impidiendo el flujo adecuado de sangre y oxígeno a la región, provocando la muerte del tejido. Es una condición que presenta un alto grado de complicaciones y altas tasas de morbimortalidad si no se realizan intervenciones tempranas. Entre las complicaciones más frecuentes se encuentran las que se producen como consecuencia del proceso inflamatorio desencadenado por la zona infartada, especialmente la formación de edemas. La hidrocefalia obstructiva, seguida de la compresión del tronco encefálico con la consiguiente muerte cerebral, es el resultado más temido de esta afección médica. Consideraciones finales: La craniectomía descompresiva es una técnica quirúrgica capaz de reducir eficazmente los efectos hipertensivos intracraneales, liberando espacio para el edema cerebral formado tras la isquemia, pudiendo mejorar la supervivencia funcional en pacientes víctimas de este raro subtipo de ictus.

Palabras clave: Ictus, Isquemia, Fosa posterior, Craniectomía descompresiva.

\section{INTRODUÇÃO}

As doenças cardiovasculares são a principal causa de morte no mundo. No grupo dessas doenças está o Acidente Vascular Encefálico (AVE) isquêmico ou hemorrágico. Os AVEs isquêmicos são mais comuns, correspondendo a $80 \%$ dos casos, enquanto apenas $20 \%$ são hemorrágicos. Aproximadamente um quinto de todos os AVEs ocorrem em vasos da circulação posterior. Uma isquemia cerebelar extensa pode cursar com efeito de massa e aumentar significativamente o risco de hidrocefalia e compressão do tronco encefálico. A técnica da craniectomia descompressiva realizada de forma precoce tem como alvo esses pacientes, a fim de reduzir as complicações letais desse quadro (MOON JW e HYUN DK, 2017).

Embora a estimativa do prognóstico seja difícil, pacientes com infarto cerebelar tendem a ter um resultado mais favorável do que pacientes com outros subtipos de AVE. O momento da cirurgia é primordial nesses pacientes. Enquanto alguns autores argumentam que a terapia cirúrgica deve ser considerada apenas quando há uma redução significativa no nível de consciência, outros tendem a agir de forma mais agressiva, uma vez que sinais clínicos ou neurorradiológicos de deterioração podem ser inespecíficos ou detectados tardiamente (PALLESEN LP, et al., 2019).

A craniectomia com ou sem inserção de Derivação Ventricular Externa (DVE) é um procedimento eficiente para prevenção de lesões maciças posteriores a edema devido a acidente vascular encefálico de circulação posterior. A craniectomia descompressiva é a remoção de um segmento do crânio grande o suficiente para reduzir a pressão intracraniana refratária e manter a complacência cerebral com o objetivo de prevenir a lesão de outras estruturas e a deterioração neurológica (BROWN DA e WIJDICKS EFM, 2017).

Este trabalho teve por objetivo fornecer uma ampla abordagem para o enfrentamento dessa condição e de suas principais complicações em diferentes cenários assistenciais, comprovando a eficácia da craniectomia descompressiva no prognóstico de um paciente vítima de AVE isquêmico de fossa posterior, com enfoque no seu tempo de realização.

\section{REVISÃO BIBLIOGRÁFICA}

\section{Antecedentes históricos da craniectomia descompressiva}

Por razões supersticiosas ou terapêuticas, nos anos de 4000 aC, os antigos incas trepanaram o crânio, sendo esta a primeira cirurgia craniana registrada na história da humanidade. No Antigo Egito e na Grécia Antiga também foram encontradas evidências de tratamento para Traumatismo Crânio-Encefálico (TCE) por meio de descompressão cirúrgica. Mas, foi Gallen quem introduziu a anatomia craniana e descreveu indicações mais precisas para a prática da descompressão, sendo indicada para casos de lesões cerebrais traumáticas, com em hematomas e fraturas (MOON JW e HYUN DK, 2017). 
No ano de 1984, a craniectomia descompressiva foi descrita pela primeira vez como um procedimento. Durante o século XIX, essa técnica foi utilizada por diversos neurocirurgiões. Já em no súculo XX, a craniectomia foi proposta por Kocher para pacientes aqueles pacientes com aumento da Pressão Intracraniana (PIC). Kocher Harvey Cushing realizou estudos utilizando a craniectomia no tratamento de outros distúrbios cerebrais, como em casos de edema cerebral pós-traumático, malformações vasculares e tumores cerebrais (NEUFELD EA, et al., 2019).

Em razões de resultados clínicos desfavoráveis em pós-operatórios, a craniectomia descompressiva não era bem aceita até o final dos anos 1970. Os estudos sobre essa técnica tiveram avanços significativos somente na década de $80 \mathrm{e}$, atualmente, vem aumentando de forma expressiva o uso da craniectomia como tratamento para diversas patologias cerebrais. Em 2009, foram realizados alguns estudos que mostraram a cirurgia de descompressão para afecções cerebrais que ocupam espaço, reduzindo significativamente a letalidade e descreveram os desfechos favoráveis desse tratamento dentro de 48 horas do início do AVE em pacientes com infartos malignos (ROSSINI Z, et al., 2019)

\section{Tratamento do AVE isquêmico de fossa posterior}

Os AVEs isquêmicos e hemorrágicos podem ser tratados de forma conservadora ou cirúrgica a depender da gravidade e extensão. Os consensos médicos para tratamento conservador em geral incluem: proteção das vias aéreas, principalmente em casos de rebaixamento da consciência, oxigenação adequada (geralmente > 94\%), monitorização e controle da pressão arterial e da frequência cardíaca, controle dos níveis de glicêmicos, medição de temperatura, prevenção de Trombose Venosa profunda (TVP) e Tromboembolismo Pulmonar (TEP) com medicações específicas. O nível de consciência deve ser avaliado rigorosamente e exames de imagem realizados a cada 48 horas para acompanhamento ou imediatamente em casos de sinais clínicos de deterioração neurológica (PALLESEN LP, et al., 2019).

Alguns médicos preconizam que pacientes alertas e clinicamente estáveis devem ser tratados de forma conservadora e observados minuciosamente utilizado escalas clínicas, como a Escala de Coma de Glasgow (ECG) para acompanhamento do nível de consciência além de neuroimagem repetida. Outros recomendam intervenção cirúrgica precoce ou preventiva, uma vez que sinais de deterioração são inespecíficos e parâmetros neurorradiológicos são incertos ou podem ser detectados tarde demais. Esses pacientes devem ser idealmente admitidos em uma UTI ou em uma unidade de AVE, onde intervenções neurocirúrgicas podem ser realizadas 24 horas por dia. Pacientes sonolentos e gravemente enfermos não devem, em circunstância alguma, serem admitidos numa ala geral (NEUGEBAUER $\mathrm{H}$, et al., 2013).

A cirurgia tem sido amplamente aceita entre neurocirurgiões e neurologistas como tratamento de escolhaem casos de efeito de massa extenso, causado por edema cerebral, uma vez que as estratégias de tratamento conservador geralmente não são bem-sucedidas nesses quadros. No entanto, o momento no qual iniciar o tratamento cirúrgico e quais fatores devem desencadear a intervenção ainda não são bem definidos. Além disso, o grande número de procedimentos disponíveis - ventriculostomia, cirurgia descompressiva suboccipital de fossa posterior com ou sem ressecção de tecido necrótico ou uma combinação de ambos não foram testados ou comparados com outros em estudos prospectivos ou randomizados maiores para que comprovasse qual método seria o mais eficaz (CHO YJ e KANG SH, 2017).

A neuroimagem deve ser realizada de forma precoce e imediata em pacientes cuja condição clínica se deteriora, afim de avaliar a existência ou não de infarto extenso do tronco cerebral e organizar a estratégia de tratamento. A DVE e a craniectomia são recomendadas quando o quarto ventrículo está bloqueado, impedindo a drenagem liquórica e a hidrocefalia está presente. Na grande maioria dos casos a indicação para intervenção baseia-se na deterioração clínica dos pacientes e progressão dos achados de imagem como, por exemplo, apagamento do quarto ventrículo ou compressão da cisterna quadrigeminal (NEUFELD EA, et al., 2019).

A compressão do tronco encefálico e a hidrocefalia obstrutiva podem ser revertidas ou impedidas pela craniectomia suboccipital. O embasamento para este procedimento é criar espaço para o tecido edemaciado, evitando assim a compressão do tronco cerebral e obstrução do quarto ventrículo. Também pode ser 
removido o tecido cerebelar necrótico para criar mais espaço na fossa posterior e reduzir o desenvolvimento de edema citotóxico (NEUGEBAUER H, et al., 2013).

\section{Complicações associadas ao AVE isquêmico de fossa posterior}

O efeito de massa com risco de morte é comumente encontrado em vários subtipos de isquemia cerebral e hemorragias intracranianas. Ocorre por hematomas, desenvolvimento subagudo de graves edemas cerebrais, ou ambos, herniação transtentorial ou transforaminal, sendo frequente causa de morte na maioria desses pacientes. Independentemente do subtipo de acidente vascular encefálico ou da patologia subjacente, todos os tipos de massas intracranianas representam graves riscos de alterações do tecido cerebral, compressão de estruturas saudáveis, aumento crítico da PIC e subsequentes complicações como comprometimento da Pressão de Perfusão Cerebral (PPC) (CHO YJ e KANG SH, 2017).

O edema cerebral é caracterizado como acúmulo anormal de líquido no parênquima encefálico. Existem três subtipos de edema classificados na literatura e uma combinação é encontrada no AVE isquêmico grave, como o AVE extenso de fossa posterior, com edema cerebral citotóxico desempenhando o papel principal. O edema citotóxico resulta do edema celular após privação de oxigênio, falha de substrato e energia e subsequente quebra das bombas de íons da membrana celular. Já o edema vasogênico ocorre no contexto de quebra da barreira hematoencefálica devido ao aumento da permeabilidade vascular. O edema intersticial está associado à absorção ineficiente a do líquido cefalorraquidiano (LCR) e hidrocefalia aguda. (AYLING OGS, et al., 2018).

Trata-se de uma complicação comum, mas frequentemente negligenciada, em 8 a $39 \%$ dos grandes infartos cerebelares. O momento de sua formação é variável, mas na maioria dos casos atinge seu pico entre $\circ 2^{\circ}$ e $04^{\circ}$ dia após o ictus. A fossa posterior fornece apenas um pequeno espaço para compensação e complicações podem se desenvolver rapidamente, incluindo: (1) hidrocefalia obstrutiva devido ao bloqueio do quarto ventrículo, (2) direta compressão do mesencéfalo e ponte, (3) hérnia para cima do vermis cerebelar superior através do entalhe tentorial, e (4) hérnia descendente das amígdalas cerebelares através o forame magno. Sendo as mais temidas a hidrocefalia e a compressão de tronco. À medida que a compressão continua, os pacientes perdem consciência e podem evoluir para o coma (NEUGEBAUER EJ, et al., 2016).

\section{Craniectomia descompressiva de fossa posterior}

A remoção de partes do crânio tem uma longa história desde o período neolítico, com evidências arqueológicas de sua prática em várias culturas da África, Ásia e Américas (BROWN DA e WIJDICKS EFM, 2017). Por craniectomia descompressiva, uma proporção significativa do crânio é removida cirurgicamente, permitindo que o tecido isquêmico se desloque através do defeito cirúrgico e não para as regiões não afetadas do cérebro, evitando assim danos secundários devido ao aumento da PIC (PALLESEN LP, et al., 2019). A descompressão óssea deve ser suficientemente grande para evitar hérnia através da janela de craniectomia com compressão resultante das veias-ponte levando a um infarto venoso e desfecho paradoxalmente pior. Vários autores recomendam um diâmetro mínimo de retalho ósseo de 11 a 12 cm (BROWN DA e WIJDICKS EFM, 2017).

Estudos sugerem que a craniectomia descompressiva de fossa posterior em pacientes jovens com inserção concomitante de DVE e desbridamento do tecido infartado contribuem para menores taxas de morbimortalidade (AYLING OGS, et al., 2018). A taxa de mortalidade em pacientes não tratados com a craniectomia descompressiva de fossa posterior por edema isquêmico cerebelar foi previamente estimado em cerca de 80\% (LINDESKOG D, et al., 2019). Em geral, o tratamento cirúrgico está associado a taxas muito maiores de sobrevida comparada ao tratamento conservador. O resultado funcional geral após a cirurgia parece ser bom com 40,6 a $54,4 \%$ dos pacientes com nenhuma ou leve deficiência e 20,3 a $41,0 \%$ sofrendo de incapacidade moderada, enquanto a incapacidade grave é relatada apenas em 11,4 a $18,8 \%$ dos pacientes (NEUGEBAUER EJ, et al., 2016).

O infarto cerebelar extenso é uma condição potencialmente fatal se tratada de forma conservadora. Há evidências suficientes de que a craniectomia descompressiva de fossa posterior realizada de forma precoce pode salvar as vidas desses pacientes, aliviando o efeito de massa regional e suas complicações associadas, 
reduzindo a taxa de mortalidade e favorecendo a recuperação com remissão praticamente completa do quadro neurológico (LINDESKOG D, et al., 2019).

Estudos disponíveis mostraram que o tratamento conservador sozinho está associado a uma taxa de mortalidade de $42,9 \%$, aumentando para uma taxa de até $85 \%$ nos pacientes que progrediram para coma. 0 tratamento cirúrgico está associado a uma maior taxa de sobrevida: 81,6\% para pacientes tratados apenas com DVE, $76,8 \%$ para aqueles tratados apenas com craniectomia descompressiva e $77,5 \%$ para aqueles tratados com ambas as técnicas. Como o pico do edema ocorre entre o $2^{\circ}$ e $4^{\circ}$ dias após o episódio, o ideal é que a craniectomia precoce de fossa posterior seja realizada em até 48 horas do evento, independente da deterioração clínica ou de exames de imagem, a fim de prevenir de forma mais segura a ocorrência de complicações garantindo um desfecho favorável a esses pacientes (NEUGEBAUER EJ, et al., 2016).

\section{Técnica cirúrgica para realização da craniectomia descompressiva infratentorial/suboccipital}

As técnicas para realização da craniectomia infratentorial ou suboccipital, como também é chamada, não são tão bem definidos quando comparadas às descrições para a craniectomia supratentorial, utilizada como tratamento para isquemias em outras regiões do cérebro. Alguns detalhes podem variar a depender da referência utilizada, como o tamanho retirado do crânio, a lateralidade e a necessidade ou não de ressecção do arco posterior do atlas. Contudo, o objetivo básico dessa cirurgia será sempre o mesmo: a descompressão acima do cerebelo edemaciado para liberar espaço (AYLING OGS, et al., 2018)

Esse procedimento é comumente realizado com o paciente em decúbito ventral ou semipronado lateralmente. É realizada uma incisão linear na linha média que se estende desde o ínion até a coluna cervical superior. Em sequência, as camadas musculares são separadas da linha média no plano avascular, deixando o crânio suboccipital, a membrana atlanto-occipital e o arco posterior do atlas expostos. É realizada uma ampla craniectomia que se estende para o forame magno. Com o objetivo de evitar a herniação tonsilar, devese seguidamente remover o arco posterior do atlas. Geralmente a dura-máter é aberta em forma de $\mathrm{Y} e$ realiza-se uma duroplastia de expansão (BEEZ T, et al., 2019).

\section{Complicações associadas à craniectomia descompressiva}

Após a realização de uma cirurgia de craniectomia descompressiva, as duas principais complicações possíveis de ocorrer são:expansão de contusões e sangramentos. As expansões, em sua grande maioria, ocorrem de forma aguda após a cirurgia e pode levar a um quadro de deterioração clínica, com necessidade depermanência hospitalar prolongada, podendo até mesmo levar o paciente a óbito. Uma possível causa para isso é a ideia de que o efeito hemostático/tamponamento é perdido na remoção do osso e, juntamente com a redução da PIC, a expansão é facilitada principalmente no lado ipsilateral à cirurgia. Outras consequências possíveis envolvem o surgimento ou aumento do tamanho de hematomas, como hematomas extradurais e hematomas subdurais agudos, ocorrendo, principalmente, no local da fratura (GOPALAKRISHNAN MS, et al. 2018).

Após a realização da cirurgia de craniectomia podem surgir algumas complicações. Dentre essas complicações está a hérnia cerebral externa, que ocorre em cerca de um quarto dos casos. Ela pode ser causada principalmente pelo edema induzido pela reperfusão cerebral e aumento do gradiente hidrostático dos capilares sanguíneos. Torção de veias de drenagem e protuberância cerebral nas bordas da craniectomia pode ocorrer devido ao edema cerebral e acabar causando congestão venosa, infartos, herniação adicional e lacerações do parênquima cerebral, agravando ainda mais quadro (AVANALI R, et al., 2019).

Quando há, sob o retalho do couro cabeludo cavado, uma pressão intracraniana subatmosférica negativa pode acabar ocorrendo a hérnia paradoxal, que é uma complicação incomum. Isso pode fazer com que o cérebro hernie quando procedimentos como punção lombar remoção de líquido cefalorraquidiano (LCR), derivação ventriculoperitoneal ou drenagem de fluido subdural são realizados. Para reverter esse quadro de herniação tem sido muito utilizada a hidratação intravenosa abundante (BEEZ T, et al., 2019).

$\mathrm{Na}$ ferida após craniectomia descompressiva podem ocorrer complicações como deiscência, ulceração ou necrose. $O$ aumento da probabilidade de lesão da artéria temporal superficial durante a cirurgia de 
emergência, assim como o tamanho do retalho de couro cabeludo, são fatores quepredispõem as bordas da ferida à isquemia nas áreas parietal posterior e temporal e essa isquemia pode ser agravada pela pressão da protuberância do cérebro. Quando o ferimento é rompido, o cérebro subjacente, exposto, ferido ou isquêmico é muito vulnerável a complicações infecciosas graves. A chance de ruptura do retalho isquêmico pode ser reduzida através da preservação meticulosa da artéria temporal superficial e da limitação da extensão posterior do retalho a não mais do que $5 \mathrm{~cm}$ atrás da orelha (GOPALAKRISHNAN MS, et al. 2018).

\section{CONSIDERAÇÕES FINAIS}

O AVE de fossa posterior é um subtipo incomum de isquemia cerebral e, quando ocorre, se não forem realizadas intervenções adequadas em tempo hábil pode trazer graves sequelas ao indivíduo e inclusive levar à óbito por compressão de tronco encefálico. A craniectomia descompressiva é uma intervenção cirúrgica baseada em princípios fisiopatológicos sólidos e, quando aplicada de forma adequada em pacientes selecionados, melhora as chances de sobrevida e o resultado funcional dos indivíduos acometidos. Deve ser feita idealmente nas primeiras 48 horas após o ictus, mesmo na ausência de deterioração neurológica, uma vez que essas podem aparecer tardiamente. Dentre as possíveis complicações do procedimento estão: contusões, sangramentos, infecção de ferida operatória e hérnia cerebral.

\section{REFERÊNCIAS}

1. AVANALI R, et al. Role of Decompressive Craniectomy in the Management of Cerebral Venous Sinus Thrombosis. Frontiers Neurology, 2019; 10:511.

2. AYLING OGS, et al. Suboccipital Decompressive Craniectomy for Cerebellar Infarction: A Systematic Review and Meta-Analysis. World Neurosurgery, 2018; 110:450-459.

3. BEEZ T, et al. Decompressive craniectomy for acute ischemic stroke. Critical Care, 2019; 23:209.

4. BROWN DA, WIJDICKS EFM. Decompressive craniectomy in acute brain injury. Handbook of Clinical Neurology, 2017; 140:299-318.

5. CHO YJ, KANG SH. Review of Cranioplasty after Decompressive Craniectomy. Korean Journal of Neurotrauma, 2017; 13(1):9-14.

6. GOPALAKRISHNAN MS, et al. Complications of Decompressive Craniectomy. Frontiers in Neurology. $2018 ; 9: 977$.

7. LINDESKOG D, et al. Long-term functional outcome after decompressive suboccipital craniectomy for spaceoccupying cerebellar infarction. Clinical Neurology and Neurosurgery, 2019; 176:47-52.

8. MARTINS MA. Manual do residente de clínica médica. 2 ed. Barueri: Manole, 2017; 1516p.

9. MOON JW, HYUN DK. Decompressive Craniectomy in Traumatic Brain Injury: A Review Article. Korean Journal of Neurotrauma, 2017; 13(1):1-8.

10. NEUFELD EA, et al. Craniocervical junction and posterior fossa dimensions can affect need for decompressive craniectomy in posterior cranial fossa hemorrhage. World Neurosurgery, 2019; 127:e570-e577.

11. NEUGEBAUER H, et al. Space-occupying cerebellar infarction: complications, treatment, and outcome. Neurosurgical Focus, 2013; 34(5):E8.

12. NEUGEBAUER EJ, et al. Decompressive. Craniectomy for Infarction and Hemorrhage Hermann. Research Gate, 2016; 1200-1217.

13. PALLESEN LP, et al. Role of Decompressive Craniectomy in Ischemic Stroke. Frontiers in Neurology, 2019; 9(9):1119.

14. ROSSINI Z, et al. The History of Decompressive Craniectomy in Traumatic Brain Injury. Frontiers Neurology, 2019; $10: 458$.

15. SREDNI S, SREDNI RAF. Cefaleia causada por dissecção de artéria vertebral: relato de caso. Revista dor, 2012; 13(4):382-384. 